\title{
Surface-induced orientational phase transition in a lyotropic liquid crystal observed by nonlinear optical techniques
}

\author{
I. H. Bechtold, ${ }^{1,3}$ S. L. Gómez, ${ }^{3}$ J. J. Bonvent, ${ }^{2}$ E. A. Oliveira, ${ }^{3}$ J. Hohlfeld, ${ }^{1}$ and Th. Rasing ${ }^{1}$ \\ ${ }^{1}$ NSRIM, University of Nijmegen, Toernooiveld 1, 6525 ED Nijmegen, The Netherlands \\ ${ }^{2}$ Universidade de Mogi das Cruzes, Avenida Dr. Cândido Xavier de Almeida Souza 200, CEP 08780-911, Mogi das Cruzes, \\ São Paulo, Brazil \\ ${ }^{3}$ Instituto de Física, Universidade de São Paulo, P.O.box. 66318, São Paulo, CEP 05315-970, Brazil
}

(Received 4 September 2003; published 4 June 2004)

\begin{abstract}
We have observed a phase transition from a uniaxial to a biaxial nematic phase in a lyotropic liquid crystal as a function of decreasing film thickness. The results, obtained by optical second-harmonic generation experiments in a wedged cell geometry, are supported by additional Z-scan measurements and can be interpreted by wall-induced ordering effects.

DOI: 10.1103/PhysRevE.69.061707

PACS number(s): 61.30.Hn, 42.65.Ky, 42.70.Df
\end{abstract}

\section{INTRODUCTION}

The study of confinement effects in liquid crystal (LC) materials is not only of fundamental interest but is also highly relevant for the large variety of LC applications, for example, in liquid crystal displays. These effects have therefore been well studied for thermotropic LC's where they have been shown to lead to changes in phase, phase transition temperatures, and dynamics [1-4].

In contrast, not so much is known about confinement effects in lyotropic liquid crystals (LLC's) that are obtained by the dispersion of amphiphilic molecules in a solvent (usually water) that self-assemble in anisotropic aggregates called micelles, despite the relevance of these materials for many fields such as biology, detergents, oil recovery, etc. Only very recently it was observed that drastic changes in the dynamical behavior are induced when the thickness of the sample is decreased, suggesting a possible nematic uniaxial-biaxial phase transition [5].

It is well known that lyotropic systems can present two nematic uniaxial phases (calamitic $N_{c}$ and discotic $N_{d}$ ) and one biaxial nematic phase $N_{b x}$. X-ray diffraction studies have shown that in the three nematic phases, the micellar aggregates have approximately the same biaxial shape, with dimensions around $8.5 \times 5.5 \times 2.6 \mathrm{~nm}^{3}$ [6-8]. Figure 1 illustrates a biaxial micelle and its symmetry axes: $\mathbf{a}, \mathbf{b}$, and $\mathbf{c}$. Free rotations around the $\mathbf{a}$ and $\mathbf{b}$ axes characterize the $N_{c}$ and $N_{d}$ nematic phases, respectively, whereas in the $N_{b x}$ phase there are only small amplitude rotations around the three symmetry axes. Therefore, the phase transitions differ from each other mostly by orientational fluctuations around the symmetry axes of the micelles.

In this paper we show that the interactions with a confining wall indeed lead to a surface-induced phase transition in the bulk of a thin LLC film. Using the nonlinear optical technique of second-harmonic generation (SHG) we have observed a phase transition from a uniaxial to a biaxial nematic phase in a confined LLC film below a thickness of about $80 \mu \mathrm{m}$. This interpretation is supported by additional Z-scan measurements and explained by the restrictions that the confining walls impose on the micelle fluctuations.
The theoretical background for SHG studies of LC has been reported in detail in previous papers [9]. Here we will only summarize the most essential part for this study. The source of the output SH signal, at frequency $2 \omega$, is governed by the nonlinear polarization $\mathbf{P}(2 \omega)$ induced by the incoming laser field $\mathbf{E}(\omega)$, which can be written as

$$
\mathbf{P}(2 \omega)=\chi_{\text {eff }}^{(2)}(2 \omega): \mathbf{E}(\omega) \mathbf{E}(\omega),
$$

where the effective nonlinear susceptibility tensor $\chi_{\text {eff }}^{(2)}$ includes dipole and quadrupole contributions, respectively. The contributing nonzero tensor elements of $\chi_{\text {eff }}^{(2)}$ depend on the symmetry of the material studied [10]. In centrosymmetric materials (such as the present LC phases), the dipolar contribution is only allowed at the symmetry breaking interfaces. At normal incidence, the only contribution can come from anisotropic terms such as $\chi_{x x x}$ and $\chi_{y y y}$. Given the thickness range of the present experiments $(30-140 \mu \mathrm{m})$ these terms can safely be ignored here $[9,10]$.

Therefore, the SHG signal is expected to originate only from the bulk and will be proportional to the film thickness for such dilute system where absorption at $\omega$ and $2 \omega$ can be ignored. The responsible quadrupole term will depend on the nonlinear polarizability of the micelles and their distribution function.

For amphiphilic molecules, it has been reported that their nonlinear optical response is mostly due to the asymmetry of the $\pi^{-}$electron group with a hydrophilic head on one side

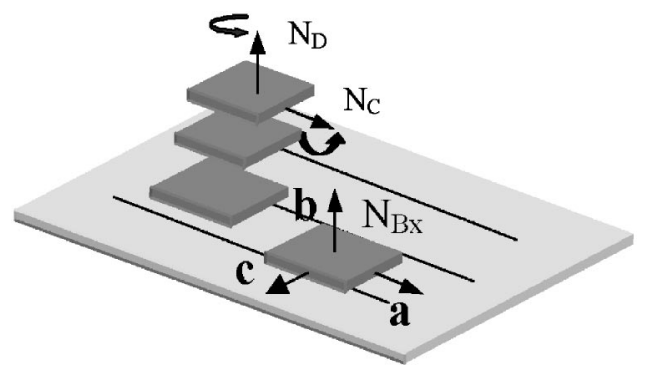

FIG. 1. Schematic representation of a micelle, indicating the orientational axes $\mathbf{a}, \mathbf{b}$, and $\mathbf{c}$. 


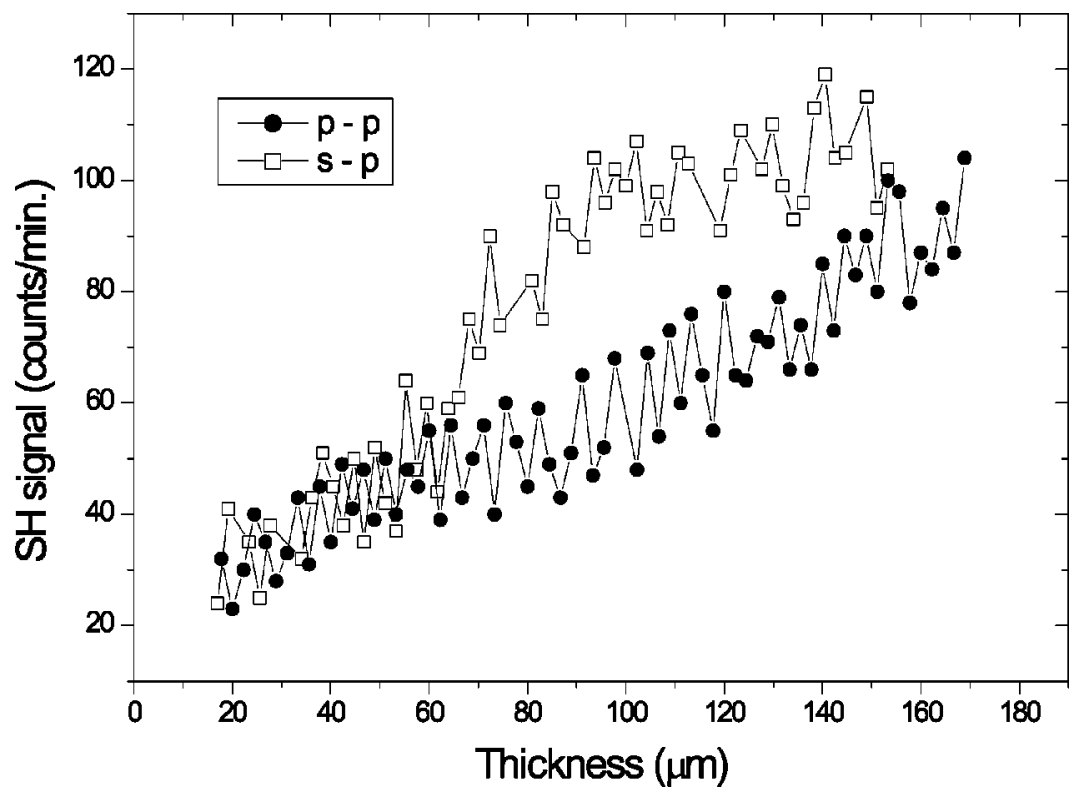

FIG. 2. SH signal as a function of the LLC sample thickness for $p-p$ and $s-p$ polarization combinations. The translation direction coincides with the uniaxial direction.

and a hydrocarbon chain on the other side [11]. However, the LLC system is composed of anisotropic aggregates of amphiphilic molecules, where the polarizability is not well known. More information on this can fortunately be obtained by $Z$-scan measurements (see below )[12].

\section{EXPERIMENT}

The LLC mixture used was composed of potassium laureate $(35.3 \mathrm{wt} \%)$, decylammonium chloride $(4.0 \mathrm{wt} \%)$, and water $(60.7 \mathrm{wt} \%)$, which exhibits the following phase sequence as determined by birefringence measurements: $H_{\alpha}-17.0^{\circ} \mathrm{C}-N_{c}-36.5^{\circ} \mathrm{C}-N_{b x}-39.0^{\circ} \mathrm{C}-N_{d}$, where $H_{\alpha}$ is a hexagonal phase. The LC material was introduced by capillary action into cells made from Poly-methyl-methacrylate (PMMA) spin coated flat glass plates that were unidirectionally rubbed to induce an easy axis. For the thickness dependence measurements, wedged cells were built with a $200 \mu \mathrm{m}$ thick spacer at only one side, providing a range of $20-160 \mu \mathrm{m}$ with an accuracy of $\approx 5 \mu \mathrm{m}$. The alignment was further improved by applying a strong magnetic field $(\approx 10 \mathrm{kG})$, parallel to the rubbing direction during $\approx 1 \mathrm{~h}$. The quality of the alignment was checked by observing the $\mathrm{LC}$ texture in a polarizing microscope.

For the SHG experiments, we used a mode-locked Ti:sapphire laser at $787 \mathrm{~nm}$, pulse width $100 \mathrm{fs}$, repetition rate $76 \mathrm{MHz}$, beam power $40 \mathrm{~mW}$ (after the chopper), focused to $\mathrm{a} \approx 0.08 \mathrm{~mm}^{2}$ cross-sectional area. A chopper with $90 \%$ intensity reduction was used to avoid thermal effects on the sample. The SH output was detected by a photomultiplier after proper spectral filtering, followed by a photon counter system. The cell was mounted on translation and rotation systems controlled by stepping motors.

The SHG transmission measurements were performed at room temperature, i.e., in the uniaxial $N_{c}$ phase. However, the $Z$-scan measurements were performed at $35.0^{\circ} \mathrm{C}$ and $37.8^{\circ} \mathrm{C}$, with the LLC sample in the $N_{c}$ and $N_{b x}$ phases, respectively.

Details of the Z-scan setup are described elsewhere [12]. Briefly, we used a cw Millennia II (Spectra Physics) laser, $\lambda=532 \mathrm{~nm}$, illuminating the sample with pulses of $33 \mathrm{~ms}$ via a mechanical chopper. The on-axis intensity in the far field was measured with a photodiode. The sample was in a $200 \mu \mathrm{m}$ thick cell aligned in a $10 \mathrm{kG}$ magnetic field. The temperature of the sample was controlled with a resolution of $\pm 0.1{ }^{\circ} \mathrm{C}$. In the $Z$-scan technique, a polarized laser beam, propagating in the $z$ direction, is focused to a narrow waist. The sample is moved along the $z$ direction through the focal point and the on-axis-transmitted intensity is measured in the far field as a function of the $z$ position of the sample. The focal volume varies with the position of the sample and can be estimated by $\pi d\left(w_{z}\right)^{2}$, where $d$ is the thickness and $w_{0}$ $\approx 20 \mu \mathrm{m}$ is the beam radius at the $z_{0}$ position.

\section{RESULTS AND DISCUSSIONS}

The intensity of the SHG signal as a function of the sample thickness is plotted in Fig. 2. The $p-p$ polarization shows a linear increase of the SHG signal with increasing thickness whereas, for the $s-p$ polarization, a discontinuous change of the SHG signal is observed around $80 \mu \mathrm{m}$. Here we define as $p$ and $s$, the polarization parallel and perpendicular to the uniaxial direction, respectively. The linearly increasing $p-p$ signal is consistent with the bulk origin of the SHG and indicates that there are no orientational changes in the direction of the sample orientation. The change in the $s-p$ signal suggests a change in the orientational order of the micelles along the axis perpendicular to the alignment direction. It is worth to underline that the measurements were repeated with different cells and this behavior is very reproducible. 

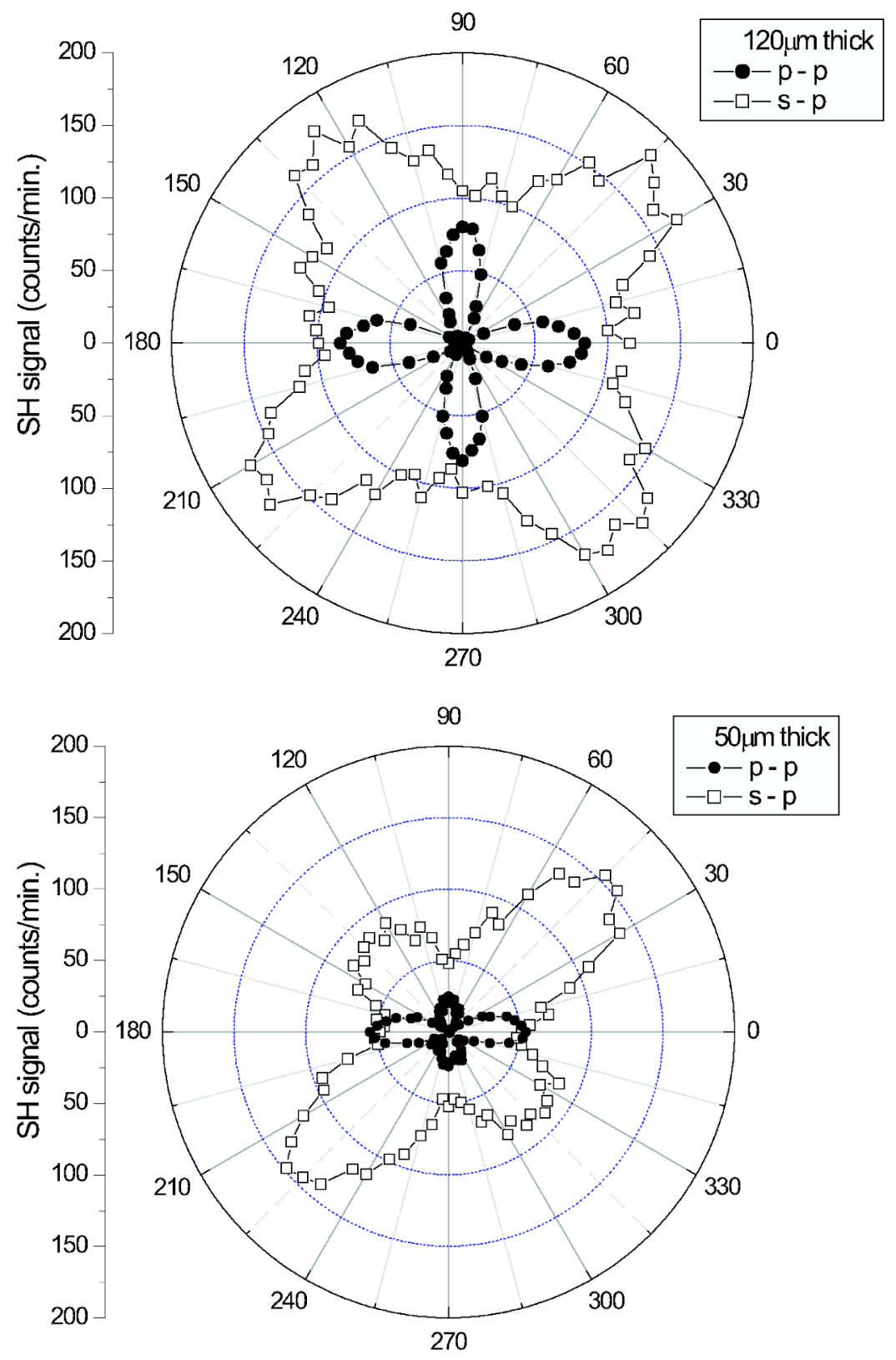

FIG. 3. SH azimuthal orientational distribution of the aligned LLC sample, measured for $p-p$ and $s-p$ polarization combinations. (a) $120 \mu \mathrm{m}$ thick; (b) $50 \mu \mathrm{m}$ thick. $\Phi=0$ coincides with the director orientation.

To further investigate the origin of the sudden signal change near $80 \mu \mathrm{m}$ we investigated the azimuthal orientational distribution for $120 \mu \mathrm{m}$ and $50 \mu \mathrm{m}$ thick cells, as is shown in Figs. 3(a) and 3(b), respectively. Although the sample is supposed to be in the $N_{c}$ phase for both thicknesses, the data in Fig. 3 show that the anisotropic SHG response exhibits polarizability components both parallel and perpendicular to the director orientation that are quite different for the two thicknesses. For the $120 \mu \mathrm{m}$ thick sample the parallel and perpendicular components have approximately the same magnitude but for the thin one $(50 \mu \mathrm{m})$, the perpendicular component is about $1 / 3$ of the parallel one. The $s-p$ polarization combination is equivalent to the $p-p$ combi- nation rotated by $45^{\circ}$, and the SH intensity is higher.

These results suggest a thickness-induced symmetry breaking. We propose that this is due to a surface-induced transition from a uniaxial nematic $N_{c}$ phase at higher thicknesses to a biaxial nematic $N_{b x}$ phase below $80 \mu \mathrm{m}$, because of the restrictions that the surface imposes on the micelle fluctuations, both around the $\mathbf{a}$ and $\mathbf{b}$ axes (see Fig. 1).

In the calamitic phase, the biaxial aggregates undergo large orientational fluctuations around the a axis. However, at the interface layers, these orientational fluctuations are restricted by the presence of the boundary surface. In addition, the surface treatment also restricts the fluctuations around the b axis, leading to a biaxial order in the surface layer [13]. 

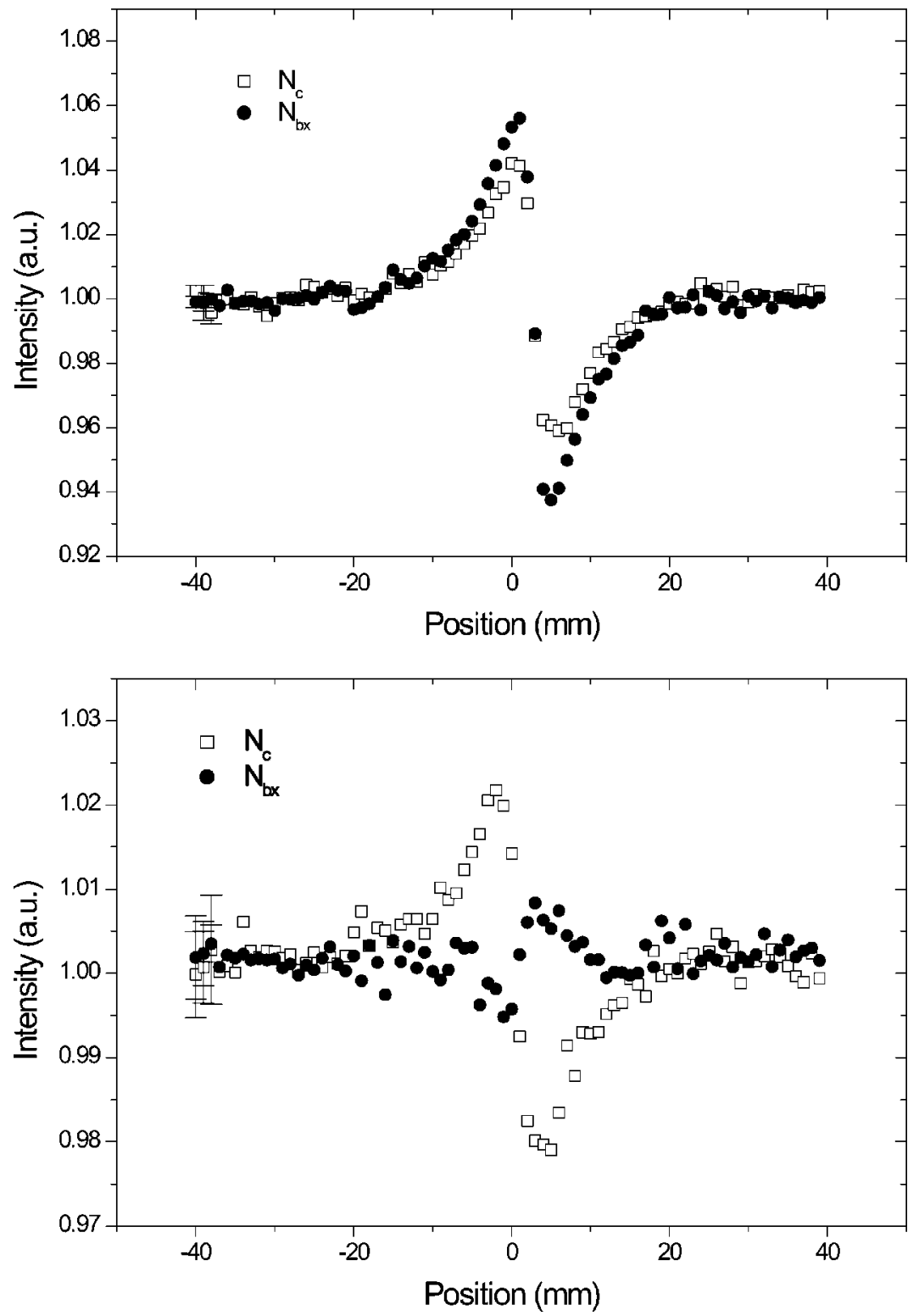

FIG. 4. Z-scan experimental curves, measured in the $N_{c}$ and $N_{b x}$ phases, with polarization of the laser beam (a) perpendicular and (b) parallel to the director orientation.

When the thickness of the sample is reduced, this order can propagate into the bulk, as was indicated with indirect measurements by previous experiments [6].

In order to confirm this hypothesis we applied the Z-scan technique on the LLC system. A cw spatially Gaussian laser beam, incident on a weakly absorbing medium, leads to a temperature profile that follows the Gaussian radial distribution of the energy. The refraction index of such a medium can be written as $n(r)=n_{o}+\delta n$ where $n_{o}$ is the linear refractive index and $\delta n$ the optical field induced refractive index change, which is proportional to the temperature coefficient of the refractive index $d n / d T$ in the millisecond time scale. Therefore, the sample behaves like an optical lens and the values of $d n / d T$ can be obtained by fitting the experimental $Z$-scan curves $[14,15]$.

We performed the $Z$-scan measurements in the $N_{c}\left(35^{\circ} \mathrm{C}\right)$ and $N_{b x}\left(37.8^{\circ} \mathrm{C}\right)$ phases, parallel and perpendicular to the director orientation, and the results are presented in Fig. 4. From the fittings of the $Z$-scan curves we obtained the values of $d n_{\|} / d T$ and $d n_{\perp} / d T$ in both nematic phases (see Table I). It has been shown that for a medium with low birefringence such as a lyotropic system $\left(\Delta n \approx 10^{-3}\right)$, the $d n / d T \propto d \alpha / d T$, where $\alpha$ is the electronic polarizability. Therefore, changes of $d n / d T$ can be attributed to changes in the $\alpha$ of the micelles $[16,17]$.

TABLE I. Values of $d n / d T$ obtained from the fitting of the experimental $Z$-scan curves.

\begin{tabular}{lcc}
\hline \hline & $d n_{\|} / d T\left(\times 10^{-3} \mathrm{~K}^{-1}\right)$ & $d n / d T\left(\times 10^{-3} \mathrm{~K}^{-1}\right)$ \\
\hline$N_{c}\left(35.0^{\circ} \mathrm{C}\right)$ & -1.30 .4 & -1.50 .4 \\
$N_{b x}\left(37.8^{\circ} \mathrm{C}\right)$ & +0.0640 .008 & -2.80 .4 \\
\hline \hline
\end{tabular}


In the $N_{c}$ phase we found that $d n_{\|} / d T$ and $d n_{\perp} / d T$ have the same sign (negative) and approximately the same value, which can be correlated with the SHG azimuthal orientational distribution for the $120 \mu \mathrm{m}$ thick cell [Fig. 3(a)] parallel and perpendicular to sample orientation. Whereas in the $N_{b x}$ phase, a drastic change is observed in the value of $d n_{\|} / d T$ (negative to positive), which is related to the inversion of the $Z$-scan curve, indicating changes in the nonlinear optical response parallel to the sample orientation. This inversion was previously observed in a $\mathrm{DeOH} / \mathrm{KL} / \mathrm{H}_{2} \mathrm{O}$ lyotropic mixture in the $N_{b x}$ phase [18]. According to Refs. $[16,17]$, such inversion is related to a sudden increase in the polarizability in that direction. Therefore, we can correlate this anisotropy to the higher $\mathrm{SH}$ intensity measured parallel to the sample orientation for the $50 \mu \mathrm{m}$ thick cell [Fig. 3(b)]. So, we can conclude that at $50 \mu \mathrm{m}$ thick the lyotropic sample is in the $N_{b x}$ phase and that the change in the SHG response below $80 \mu \mathrm{m}$ is related to a transition from a uniaxial $N_{c}$ to a biaxial $N_{b x}$ phase. Because the transition occurs for decreasing film thickness, where the influence of the confining surfaces becomes more important, we can conclude that we have indeed observed a surface-induced phase transition from a $N_{c}$ to a $N_{b x}$ phase.
In conclusion, the combination of the two nonlinear optical techniques used in this work (SHG and $Z$ scan) allowed us to confirm, with direct measurements, the existence of a surface induced nematic uniaxial to biaxial phase transition in a confined lyotropic sample below about $80 \mu \mathrm{m}$.

We showed that the SHG technique can be used to investigate the orientational order of amphiphilic structures in a LLC, when applied in a transmission geometry. It is important to underline that as far as we know, it was the first time this technique was successfully applied to lyotropic systems. From the azimuthal orientational distribution of the $\mathrm{SH}$ signal it was possible to identify the symmetry differences between the calamitic and biaxial nematic phases they exhibit.

\section{ACKNOWLEDGMENTS}

I.H.B. acknowledges FAPESP for the financial support for his research stay in Nijmegen, The Netherlands, and the University of Nijmegen for its hospitality. Part of this work was supported by the EU Network SILC. The authors are grateful to Professor A.M. Figueiredo Neto for useful discussions and to A. Van Etteger for technical support with the SHG experiments.
[1] G. S. Iannacchione and D. Finotello, Phys. Rev. Lett. 69, 2094 (1992).

[2] N. A. Clark, T. Bellini, R. M. Malzbender, B. N. Thomas, A. G. Rappaport, C. D. Muzny, D. W. Schaefer, and L. Hrubesh, Phys. Rev. Lett. 71, 3505 (1993).

[3] Liquid Crystals in Complex Geometries Formed by Polymer and Porous Networks, edited by G. P. Crawford and S. Zumer (Taylor \& Francis, London, 1996).

[4] M. M. Wittebrood, S. Stallinga, I. Musevic, and Th. Rasing, Phys. Rev. Lett. 80, 1232 (1998).

[6] A. M. Figueiredo Neto, Y. Galerne, A. M. Levelut, and L. Liébert, J. Phys. (Paris), Lett. 46, L-499 (1985).

[5] I. H. Bechtold, J. J. Bonvent, and E. A. Oliveira, Phys. Rev. E 65, 011704 (2002).

[7] A. M. Figueiredo Neto, A. M. Levelut, L. Liébert, and Y. Galerne, Mol. Cryst. Liq. Cryst. 129, 191 (1985).

[8] Y. Galerne, A. M. Figueiredo Neto, and L. Liébert, J. Chem. Phys. 87, 1851 (1987).

[9] M. B. Feller, W. Chen, and Y. R. Shen, Phys. Rev. A 43, 6778 (1991).
[10] Y. R. Shen, The Principles of Nonlinear Optics (Wiley, New York, 1984).

[11] S. G. Grubb, M. W. Kim, Th. Rasing, and Y. R. Shen, Langmuir 4, 452 (1988).

[12] S. L. Gómez, F. L. S. Cuppo, A. M. Figueiredo Neto, T. Kosa, M. Muramatsu, and R. J. Horowicz, Phys. Rev. E 59, 3059 (1999).

[13] V. L. Lorman, E. A. Oliveira, and B. Metout, Phys. Lett. A 231, 283 (1997).

[14] J. P. Gordon, R. C. C. Leite, R. S. Moore, S. P. S. Porto, and J. R. Whinnery, J. Appl. Phys. 36, 3 (1965).

[15] C. A. Carter and J. M. Harris, Appl. Opt. 23, 476 (1984).

[16] J. R. D. Pereira, A. J. Palangana, A. M. Mansanares, E. C. da Silva, A. C. Bento, and M. L. Baesso, Phys. Rev. E 61, 5410 (2000).

[17] J. R. D. Pereira, A. M. Mansanares, A. J. Palangana, and M. L. Baesso, Phys. Rev. E 64, 012701 (2001).

[18] F. L. S. Cuppo, S. L. Gómez, and A. M. Figueiredo Neto, Phys. Rev. E 67, 051711 (2003). 